\title{
Prevalencia del virus papiloma humano y factores de riesgo asociados en mujeres afiliadas al seguro de salud estatal en Posadas, Misiones (Argentina)
}

\author{
Prevalence of human papillomavirus and associated risk factors in women affiliated with \\ state health insurance in Posadas, Misiones (Argentina)
}

Graciela Beatriz Jordá1,2*, José Manuel Ramos³, Jessica Mosmann4, María Lorena Lopez², Adriana Wegert² y Cecilia Cuffini

Cátedra de Virología, Facultad de Ciencias Exactas, Químicas y Naturales, Universidad Nacional de Misiones. Argentina.
2Laboratorio Instituto de Previsión Social de Misiones. Argentina.
${ }^{3}$ Servicio de Medicina Interna del Hospital General Universitario de Alicante, Alicante (España).
${ }^{4}$ Instituto de Virología Dr. J.M. Vanella, Facultad de Ciencias Médicas. Universidad Nacional de Córdoba. Argentina.

Los autores declaran no tener conflicto de intereses.

No hubo financiación externa para la realización de este estudio.

Recibido (nueva versión): 17 de septiembre de 2019 / Aceptado: 3 de febrero de 2020

\section{Resumen}

Introducción: El virus papiloma humano (VPH) causa infecciones transmitidas sexualmente. Objetivos: Conocer la prevalencia de infecciones genitales por VPH, identificar factores clínico-epidemiológicos asociados a dicha prevalencia y determinar la frecuencia de los tipos virales. Material y Métodos: Se estudiaron muestras endo-cervicales de 505 mujeres entre 15 y 49 años, que concurrieron para estudio de exudado vaginal al laboratorio del Instituto de Previsión Social, residentes de Posadas, Misiones, entre enero de 2012 y junio de 2013. Se amplificó una sección de 450 pares de bases del genoma viral perteneciente al fragmento L1 del VPH. La detección del genotipo se realizó mediante el estudio del polimorfismo de la longitud de los fragmentos de restricción (RFLP). Resultados: La prevalencia de la portación de VPH total fue $30,7 \%$, y de éstos $71,6 \%$ correspondió a los de alto riesgo (principalmente VPH 16 [35,1\%], 58 [10,8\%] y 31 [8,1\%]). La detección del VPH fue mayor en mujeres de 15 a 24 años (OR: 1,48; IC $95 \%: 1,01-2,18$ ) y con más parejas sexuales (OR:1,81; IC $95 \%$ : $1,02-3,22)$. No hubo asociación con el embarazo, la estabilidad de la pareja, métodos anticonceptivos, edad de inicio de las relaciones sexuales ni tabaquismo. Conclusiones: Los resultados de identificación y tipificación de VPH en este estudio aportan información sobre una prevalencia elevada de VPH en mujeres sexualmente activas, así como porcentajes elevados de genotipos oncogénicos en esta región.

Palabras clave: virus papiloma humano; genotipos; prevalencia; factores de riesgo.

\begin{abstract}
Background: Human papilloma virus (HPV) is one of the most frequent sexually transmitted infections, especially among young people. Aims: To describe the prevalence of genital HPV infections, to identify clinical-epidemiological factors associated with them and to determine the frequency of viral strains. Methods: Endocervical samples were studied of 505 women between 15 and 49 years old, who attended the laboratory of the Institute of Social Security, residents of Posadas, Misiones, for the study of vaginal exudate, between January 2012 and June 2013. A 450-base pair fragment within the HPV L1 region was amplified. Genotype detection was performed through the study of the restriction fragment length polymorphism (RFLP). Results: The prevalence of total HPV carriage was $30.7 \%$, and of these $71.6 \%$ corresponded to high risk (mainly HPV 16 [35.1\%], 58 [10.8\%] and $31[8.1 \%]$ ). HPV detection was higher in women aged 15 to 24 years (OR: 1.48, 95\% CI: 1.01-2.18) and with more sexual partners (OR: $1.81,95 \% \mathrm{CI}: 1,02-3.22)$. There was no association with pregnancy, stability of the couple, contraceptive methods, age at onset of sexual intercourse, or smoking. Conclusions: The identification and typing of HPV in this study provides information regarding the high prevalence of HPV and the substantial proportion of cases with oncogenic genotypes among sexually active women in this region of Argentina.

Keywords: human papilloma virus; genotypes; prevalence; risk factors.
\end{abstract}




\section{Introducción}

$\mathrm{E}$ 1 virus papiloma humano (VPH) representa una de las infecciones de transmisión sexual más frecuentes ${ }^{1}$. Los datos publicados muestran un rango de prevalencia de 30 a $60 \%$ entre los 15 y 25 años de edad ${ }^{2}$. Se ha demostrado que el VPH favorece el desarrollo de lesiones pre-neoplásicas o neoplasias muco-cutáneas ${ }^{3}$.

El cáncer cervical es el cuarto tipo de cáncer más común en mujeres de todo el mundo y el primero en países en desarrollo. La Organización Mundial de la Salud reporta una incidencia mundial de cáncer cervical de aproximadamente 570.000 casos invasores diagnosticados por año $0^{4}$. En el año 2018, más de 72.000 mujeres fueron diagnosticadas de cáncer cérvico-uterino y casi 34.000 fallecieron por esta enfermedad en la región de las Américas ${ }^{5}$.

La Agencia Internacional de Investigación en Cáncer (IARC) informó en el año 2012 que el cáncer de cérvix, con 4.956 casos notificados en Argentina, representó 8,2\% -tercero en orden de mayor frecuencia- del total de los cánceres anuales identificados en mujeres ${ }^{6}$.

Los VPH que infectan el tracto ano-genital son alrededor de 40 tipos y han sido subdivididos en dos grupos sobre la base de su potencial oncogénico: VPH de bajo riesgo (tipos 6, 11, 42, 43, 44), comúnmente encontrados en condilomas acuminados y neoplasias intra-epiteliales de bajo grado y con mínimo riesgo de progresión maligna, y VPH de alto riesgo (tipos 16, 18, 31 y 45, entre otros), asociado a infecciones persistentes que pueden conducir al cáncer? ${ }^{7}$ El VPH 16 es el genotipo más frecuente del grupo de alto riesgo y ha sido identificado en casi la mitad de todos los cánceres de cuello uterino.

En el caso de infección por VPH de alto riesgo y bajo condiciones favorables, el genoma viral se integra al genoma de la célula hospedera, lo que es necesario para la inmortalidad de los queratinocitos ${ }^{8}$.

La mayoría de las infecciones, aun las producidas por los tipos de alto riesgo (con o sin anomalías citológicas), se mantienen por un tiempo acotado'. La duración media estimada es de 8 a 12 meses $^{1}$. Son auto-limitadas y no dejan secuelas onco-patogénicas. Sin embargo, los VPH pueden generar una infección persistente en una proporción minoritaria, aunque en valores absolutos son de importancia sanitaria por la elevada prevalencia de circulación viral en la población. La infección persistente es un factor causal necesario para la neoplasia intra-epitelial cervical. Se estima que deben transcurrir varios años (20 años aproximadamente) entre la infección inicial y el desarrollo del cáncer de cérvix ${ }^{9}$.

Al momento de redactarse este manuscrito, están disponibles y licenciadas dos vacunas contra el VPH (una tetravalente, dirigida contra VPH 6, 11, 16 y 18, y la otra, bivalente, disponible en la provincia de Misiones, dirigida contra VPH 16 y 18). Ambas son preparadas por tecno- logía recombinante, utilizando proteínas estructurales L1 purificadas, que son ensambladas en partículas símil virus (VLP). Ninguna contiene virus vivos ni ADN viral. No tienen acción terapéutica ${ }^{6}$.

\section{Objetivos}

Determinar la prevalencia de las infecciones genitales por VPH, identificar factores clínico-epidemiológicos asociados a dicha prevalencia y obtener la frecuencia de los tipos virales en mujeres sintomáticas, asintomáticas, embarazadas o no, usuarias del laboratorio de análisis clínicos del Instituto de Previsión Social (IPS).

\section{Materiales y Métodos}

Se estudiaron 505 muestras endo-cervicales de mujeres, que residían en la ciudad de Posadas, Misiones, Argentina, durante el período comprendido entre enero de 2012 y junio de 2013. Fueron incluidas todas aquellas mujeres sexualmente activas, incluso las menores de edad.

Las pacientes concurrieron al laboratorio del Instituto de Previsión Social (IPS) de la provincia de Misiones, con una solicitud de estudio de exudado vaginal por sospecha clínica de patología de tracto genital inferior o estudio de control periódico promovido por los médicos de la obra social.

El trabajo fue aprobado, por el Comité de Bioética de la Unidad Académica del Parque de la Salud de Posadas (Misiones). Todas las pacientes recibieron información oral y escrita sobre los objetivos del estudio. A las pacientes menores de edad se les exigió el consentimiento de sus padres o tutores.

A las participantes se les realizó una encuesta sobre factores clínicos (presencia y características del flujo, embarazo) y epidemiológicos (número de hijos, método anticonceptivo, edad de inicio de las relaciones sexuales, número de parejas sexuales, tabaquismo).

Se tomaron muestras del cuello uterino con hisopos de dacrón. La extracción de ADN se realizó en forma manual utilizando el kit de extracción de Machery-Nagel siguiendo las instrucciones del fabricante. Para evaluar la calidad de la muestra, integridad del ADN y/o la presencia de inhibidores, se amplificó una porción del exón III del gen de la $\beta$-actina humana ${ }^{10}$.

Para la detección del VPH se amplificó una sección de 450 pares de bases del genoma viral perteneciente al fragmento L1 mediante los cebadores consenso MY 09 y MY 11, según protocolo de Manos y cols. ${ }^{11}$. La amplificación se llevó a cabo en un volumen final de $50 \mu \mathrm{l}$ que contenía una mezcla de reacción con $0,05 \mathrm{U}$ de Taq polimerasa, $2 \mu \mathrm{M}$ de cada uno de los oligonucleótidos, 2,5 $\mathrm{mM}$ de $\mathrm{MgCl}_{2}, 0,5 \mathrm{mM}$ de cada uno de los cuatro dNTPs, tampón para la enzima Taq polimerasa y $10 \mu \mathrm{l}$ del templado. 
La reacción de polimerasa en cadena (RPC) se llevó a cabo utilizando un termociclador compacto Labnet, modelo MultiGene ${ }^{\mathrm{TM}}$ II Personal Thermal Cycler, con un programa que incluía una desnaturalización inicial a $94^{\circ} \mathrm{C}$ durante tres minutos, seguido de 35 ciclos de un minuto a $94^{\circ} \mathrm{C}$, un minuto a $50^{\circ} \mathrm{C}$ y un minuto a $72^{\circ} \mathrm{C}$, con una extensión final de cinco minutos a $72^{\circ} \mathrm{C}$. Como control negativo de la RPC se utilizó la misma mezcla de reacción sometida a las mismas condiciones con el agregado de agua ultra pura y como control positivo genoma de la línea celular CaSki infectada persistentemente con VPH16. Los productos de amplificación se corrieron por electroforesis en gel de agarosa al 1,5\% teñido con bromuro de etidio, a 90 voltios durante 20 minutos y se observaron por exposición a luz ultravioleta en el transiluminador.

La detección del genotipo de VPH se realizó mediante el estudio del polimorfismo de la longitud de los fragmentos de restricción (RFLP). A partir del fragmento amplificado se realizó la digestión enzimática con las siguientes endonucleasas de restricción: Bam H1, DdeI, HaeIII, HinfI, PstI, RsaI y Sau 3 AI, de acuerdo a la técnica propuesta por Bernard y cols ${ }^{12}$. Se preparó un tubo para cada enzima con $10 \mu \mathrm{l}$ de agua. Se adicionó $6,5 \mu \mathrm{ldel}$ producto de la RPC, $2 \mu 1$ de tampón y 1,5 $\mu 1$ de enzima de restricción. Se incubó $2-4 \mathrm{~h}$ a $37^{\circ} \mathrm{c}$. Se analizaron los patrones de corte tipo viral específicos producidos por las enzimas mencionadas en un gel de agarosa al $2 \%$ en tam- pón tbe $1 \mathrm{x}$ se sembró un control sin digerir del producto de 450 pb, se corrió 60 min a 60 voltios y se compararon los patrones contra patrones de digestión conocidos para su identificación ${ }^{12}$.

Los casos positivos, en los que no se pudo determinar el genotipo por la metodología empleada, fueron catalogados como VPH-x. La detección de dos o más secuencias de ADN del VPH detectadas simultáneamente en una misma muestra se consideró una infección múltiple.

\section{Análisis estadístico}

El análisis estadístico se efectuó con la prueba de $\chi^{2}$, con el programa SPSS versión 21.0. Se consideraron estadísticamente significativos los valores con $p<0,05$. Los resultados de prevalencia se mostraron con un intervalo de confianza (IC) de 95\%. La asociación entre variables se midió mediante el cálculo de Odds Ratio (OR) con un IC de $95 \%$.

\section{Resultados}

Se estudiaron 505 mujeres cuyo rango de edad estuvo comprendido entre 15 y 49 años de edad, con una media de 28,4 años (DE 8,2). Se detectó VPH en 155 de ellas, lo que representa una prevalencia de $30,7 \%$.

En la Tabla 1 se analizan los factores clínicoepidemiológicos considerados de interés y de probable

\begin{tabular}{|c|c|c|c|c|c|}
\hline & $\begin{array}{c}\text { Total } \\
\mathbf{n}=\mathbf{5 0 5}\end{array}$ & $\begin{array}{l}\text { VPH (+) } \\
n=155\end{array}$ & $\begin{array}{c}\text { VPH (-) } \\
n=350\end{array}$ & OR (IC 95\%) & Valor $p$ \\
\hline Edad media (DE) & $28,4(88,2)$ & $27,4(7,9)$ & $28,8(8,4)$ & $0,98(0,95-1,00)$ & 0,067 \\
\hline \multicolumn{6}{|l|}{ Rango de edad, n (\%) } \\
\hline $25-49$ años & $304(60,2)$ & $83(53,5)$ & $221(63,1)$ & 1 & \\
\hline 15-24 años & $201(39,8)$ & $72(46,5)$ & $129(36,9)$ & $1.48(1.01-2.18)$ & 0,042 \\
\hline Embarazo, n (\%) & $106(21,0)$ & $29(18,7)$ & $77(22,0)$ & $0,82(0,50-1,31)$ & 0,402 \\
\hline Síntomas, n (\%) & $265(52,5)$ & $78(50,3)$ & $187(53,4)$ & $0,88(0,60-1,28)$ & 0,519 \\
\hline Número embarazos, media (DE) & $1,1(1,3)$ & $1,0(1,3)$ & $1,2(1,3)$ & $0,86(0,74-1,01)$ & 0,065 \\
\hline Número de hijos, media (DE) & $0,8(1,1)$ & $0,7(1,1)$ & $0,9(1,1)$ & $0,88(0,73-1,05)$ & 0,167 \\
\hline \multicolumn{6}{|c|}{ Edad de inicio de relaciones sexuales, $\mathrm{n}(\%)$} \\
\hline Entre 19 y 33 años & $140(27,7)$ & $41(26,5)$ & $99(28,3)$ & 1 & \\
\hline Entre 13 y 18 años & $365(72,3)$ & $114(73,5)$ & $251(71,7)$ & $1,09(0,71-1,68)$ & 0,671 \\
\hline \multicolumn{6}{|l|}{ Número de parejas sexuales, n (\%) } \\
\hline 1 & $108(21,4)$ & $25(16,1)$ & $83(23,7)$ & 1 & \\
\hline $2-5$ & $267(52,9)$ & $84(54,2)$ & $183(52,3)$ & $1,52(0,90-2,55)$ & 0,108 \\
\hline$>5$ & $130(25,7)$ & $46(29,7)$ & $84(24,0)$ & $1,81(1.02-3,22)$ & 0,040 \\
\hline Uso de anticonceptivos orales, n (\%) & $188(37,2)$ & $61(39,4)$ & $127(36,4)$ & $1,13(0,77-1,78)$ & 0,511 \\
\hline Uso de preservativo, n (\%) & $90(17,8)$ & $30(19,4)$ & $60(17,1)$ & $1,16(0,71-1,89)$ & 0,549 \\
\hline Tabaquismo, n (\%) & $74(14,7)$ & $17(11,0)$ & $57(16,3)$ & $0,63(0,36-1,13)$ & 0,119 \\
\hline
\end{tabular}


asociación a la infección por VPH. La prevalencia de VPH en mujeres entre 15 y 24 años fue de $35,8 \%$ (72/201), mayor que en mujeres entre 25 y 49 años (27,3\% [83/304]) (OR: 1,48; IC 95\%: 1,01-2,18). Se encontró asociación con el mayor número de parejas sexuales (OR: 1,81; IC 95\%: 1,02-3,22). Al analizar la asociación de VPH con métodos anticonceptivos, embarazo, edad de inicio de las relaciones sexuales y tabaquismo, no se observaron diferencias estadísticamente significativas.

Se realizó la técnica de RFLP a las 155 muestras positivas, logrando la genotipificación solamente en 74 de ellas (47,7\%). En 52,3\% (81/155) de los casos positivos para VPH no se logró identificar secuencias patrones a través del método de detección utilizado (VPH-x). En $71,6 \%(53 / 74)$ se detectó secuencias de ADN de VPH de alto riesgo, así como en 28,4\% (21/74) secuencias de VPH de bajo riesgo. La infección múltiple por VPH estaba presente en $21,6 \%$ (16/74) de las muestras genotipificadas.

La distribución de los 16 tipos de VPH identificados y su frecuencia se observa en la Tabla 2. Los cinco tipos de VPH de bajo riesgo identificados fueron: 6, 11, 54, 57 y 61 y los once tipos de VPH de alto riesgo: 16, 18, 31, $33,35,45,53,58,59,66$ у 68 .

\section{Discusión}

Es difícil establecer estimaciones referidas a la cantidad de mujeres portadoras de infecciones ocultas por VPH. Mediante técnicas altamente sensibles como hibridación molecular o RPC, puede obtenerse

\begin{tabular}{|c|c|c|c|c|c|c|}
\hline \multicolumn{4}{|c|}{ Infecciones únicas } & \multicolumn{3}{|c|}{ Infecciones múltiples } \\
\hline VPH-AR & n (\%) & VPH-BR & n (\%) & Combinación & & (\%) \\
\hline 16 & $22(29,7)$ & 6 & $5(6,8)$ & $6+x$ & 2 & $(2,7)$ \\
\hline 18 & $2(2,7)$ & 11 & $4(5,4)$ & $6+16$ & 2 & $(2,7)$ \\
\hline 31 & $5(6,8)$ & 61 & $6(8,1)$ & $16+x$ & 3 & $(4,1)$ \\
\hline 33 & $1 \quad(1,4)$ & & & $16+53$ & 1 & $(1,4)$ \\
\hline 35 & $1(1,4)$ & & & $16+54$ & 1 & $(1,4)$ \\
\hline 45 & $1(1,4)$ & & & $16+58$ & 1 & $(1,4)$ \\
\hline 53 & $2(2,7)$ & & & $18+31$ & 1 & $(1,4)$ \\
\hline 58 & $6(8,1)$ & & & $57+45$ & 1 & $(1,4)$ \\
\hline 59 & $1 \quad(1,4)$ & & & $58+x$ & 1 & $(1,4)$ \\
\hline 66 & $2(2,7)$ & & & $\begin{array}{c}61+33 \\
61+x \\
68+x\end{array}$ & & $\begin{array}{l}(1,4) \\
(1,4) \\
(1,4)\end{array}$ \\
\hline Total & $43(58,1)$ & Total & $15(20,3)$ & Total & 16( & $(21,6)$ \\
\hline
\end{tabular}

la prevalencia de VPH en la población femenina ${ }^{1}$. La prevalencia de VPH y distribución de genotipos es altamente deseable para evaluar el impacto profiláctico de las vacunas contra VPH.

Un meta-análisis de estudios publicados entre 1995 y 2009 utilizando RPC para la detección de VPH en 1.016.719 mujeres con resultados citológicos normales, informó una prevalencia global estimada para VPH de $11,7 \%$ (IC del 95\%, 11,6\% - 11,7\%); en este estudio, Latinoamérica $(16,1 \%)$ mostró una de las prevalencias más altas ${ }^{13}$. En nuestro estudio es superior la prevalencia de VPH $(30,7 \%)$, siendo similar a la encontrada por Sotlar y cols. ${ }^{14}$, en un estudio realizado en 294 mujeres en la Universidad de Türbingen, Alemania $(34,7 \%)$ u otro realizado por Camargo Pinzón y cols., en 210 mujeres en el Departamento de Salud de Cauca en Colombia donde la prevalencia fue de $39 \%{ }^{15}$.

Nuestra prevalencia fue similar a otras evaluaciones realizadas en nuestro entorno. Así, el primer estudio en Misiones, Argentina, fue realizado por Tonon y cols. ${ }^{16}$, quienes informaron una prevalencia de VPH de $43 \%$ en mujeres seleccionadas aleatoriamente y residentes en diferentes ciudades de esta provincia. Posteriormente, datos obtenidos de Badano y cols. ${ }^{17}$, mostraron una prevalencia de VPH de 33,1\% en mujeres de bajos recursos de la ciudad de Posadas, Misiones.

De las 155 muestras positivas, se logró genotipificar a 74 de ellas; esto pudo deberse a que las muestras presentaban una carga viral baja, manifestado por la débil intensidad observada en las bandas de precipitación en gel de agarosa que imposibilitaron una resolución eficaz por la técnica de RFLP. Esto es una limitante de la técnica de RFLP, resultando un método no óptimo para tipificar los VPH. También, en algunos casos, podría tratarse de virus nuevos o variantes aún no identificadas.

Los 15 tipos virales hallados en este estudio coinciden, en general, con los genotipos prevalentes en la población mundial $^{14-18}$. Los tipos de VPH 16, 18, 31, 52 y 58 se encuentran entre los 10 tipos más comunes.

En nuestra experiencia, los tipos virales de alto riesgo mostraron mayor prevalencia respecto a los de bajo riesgo y el VPH 16 fue el más frecuente coincidiendo con reportes nacionales e internacionales ${ }^{17-20}$.

En segunda frecuencia se observó el VPH 58 (10,8\%) y luego el VPH 31 (8,1\%). Es importante destacar el hallazgo del VPH 58, no habitualmente encontrado en nuestro país ${ }^{17,18}$; aunque Venezuela y cols., en un estudio realizado en la provincia de Córdoba, informaron que este genotipo comparte el segundo lugar con el VPH $18^{18}$. Clifford y cols., en un estudio que incluyó mujeres de 13 áreas de 11 países, informó que en Sudamérica el segundo tipo viral más frecuentemente encontrado fue el VPH 58 con una prevalencia de $7 \%{ }^{21}$. En un estudio en Paraguay, país colindante con Misiones, el VPH 58 también se 
observó como el segundo en orden de frecuencia $(2,4 \%)^{22}$. De acuerdo a estos resultados, se podría sugerir que el genotipo VPH 58 se incluyera en la formulación vacunal, aunque se deberían realizar estudios de persistencia para evaluar su potencial oncogénico.

Los genotipos VPH 6 y VPH 61, más frecuentemente detectados en nuestro estudio, tuvieron una prevalencia relativamente más alta $(10,8 \%)$ a lo reportado por Tonon y cols. ${ }^{16}$, para el VPH $6(8,4 \%)$ en una población semejante a la nuestra. Sin embargo, Deluca y cols. ${ }^{19}$, encontraron $5,3 \%$ de VPH 6 en un estudio de mujeres aborígenes del nordeste de Argentina. Golfetto y cols., en un estudio realizado en 325 mujeres que acudieron a un servicio público por un tamizaje cervical en el estado de Santa Catarina, Brasil, encontraron que el genotipo de bajo riesgo prevalente fue el VPH $61(3,8 \%)^{20}$.

En este estudio, la infección múltiple por VPH $(21,6 \%)$ fue menor al $27 \%$ obtenido por Miranda y cols., en San Pablo, Brasil, en una población de mujeres con citología normal, y muy superior al 1,5\% observado por Toro de Méndez y cols., en Mérida, Venezuela. Ambos estudios utilizaron una metodología molecular de detección de VPH semejante a la técnica RFLP empleada en la presente investigación ${ }^{23}$.

En este trabajo no se obtuvo asociaciones estadísticamente significativas en la población VPH positiva con factores de riesgo, tales como: alta paridad, inicio temprano de las relaciones sexuales, tabaquismo, métodos anticonceptivos, no uso de profiláctico de barrera, resultando diferente a lo observado por otros autores ${ }^{1,16,17,24}$.

Por otro lado, se encontró una asociación significativa con la edad. Nuestros hallazgos de prevalencia (35,8\%) en mujeres bajo 24 años de edad, coinciden con los reportes internacionales que estiman una prevalencia entre $30-40 \%$ en este grupo etario ${ }^{1}$. Este resultado concuerda con lo informado por Venezuela y cols. ${ }^{18}$, con Toro de Méndez y cols., y difiere de Tonon y cols., quienes hallaron una mayor frecuencia en el rango de 31-35 años ${ }^{16,23}$.

Aunque es muy probable que las infecciones por VPH detectadas en las jóvenes sean transitorias, es destacable que fueron mujeres que acudieron al laboratorio por un estudio de exudado vaginal, sin antecedentes de lesión cervical o estudio previo de Papanicolau alterado.
En general, se ha reportado en varios estudios asociación entre infección por VPH y el inicio temprano de las relaciones sexuales, aunque esto también está relacionado al comportamiento sexual y hábitos culturales de una comunidad $^{1}$. En nuestro estudio se observó que 73,5\% de las pacientes infectadas con VPH se iniciaron sexualmente entre los 15 y 18 años, pero no resultó con significación estadística. Ponce-Coello y Uyaguari Díaz realizaron un estudio en Ecuador en mujeres trabajadoras de la salud y tampoco hallaron significación estadística ${ }^{25}$.

Hemos encontrado que la prevalencia de VPH aumentó con el número de parejas sexuales y esto resultó estadísticamente significativo, coincidiendo con Medina y cols., y Badano y cols. ${ }^{17,24}$. En el grupo de mujeres con infección por VPH, que usaban el método anticonceptivo oral no se encontró asociación significativa $(\mathrm{p}=0,511)$, aunque Bosch y cols., encontraron asociación entre el uso de anticonceptivo oral y la infección con $\mathrm{VPH}^{1}$.

De las 155 mujeres con infección por VPH, 11\% manifestó el hábito de fumar y no se encontró asociación estadística significativa con este factor de riesgo. Sin embargo, Badano y cols., y Tonon y cols., hallaron al tabaquismo como un factor de riesgo en mujeres de la provincia de Misiones ${ }^{16,17}$.

El estudio tiene las limitaciones de ser unicéntrico y restringido a la población atendida en el IPS, por lo que la prevalencia encontrada en este estudio puede no ser representativa de otras poblaciones de Argentina o Latinoamérica.

Los resultados de identificación y tipificación del VPH en este estudio, aportan información sobre la persistencia de una prevalencia elevada del VPH en mujeres sexualmente activas, pertenecientes a la principal obra social de la ciudad de Posadas. El estudio destaca los porcentajes elevados de genotipos oncogénicos en esta región de Argentina, algunos de los cuales no se encuentran incluidos entre los componentes de la actual composición de la vacuna de aplicación en Misiones.

Agradecimientos: A la Facultad de Ciencias Exactas, Químicas y Naturales (UNaM) y al Instituto de Previsión Social Misiones por el uso de las instalaciones y los servicios prestados.

\section{Referencias bibliográficas}

1.- Bosch José F-Xavier, Diaz Sanchis M, Sanjosé Llongueras S, Font Marimon R, Castellsagué Piqué X, Albero Abril G, et al. Epidemiología de las infecciones por HPV: riesgo de carcinoma cervico-uterino y de otros tumores anogenitales. Nuevas opciones preventivas. En: Virus del papiloma humano y cáncer: epidemiología y prevención. Sociedad Española de Epidemiología. De Sanjosé Llongueras S, García García AM, editoras. 2006. p. 31-48. https://www.seepidemiologia.es/documents/du mmy/4monografiaVirusPapilomaYCancer.pdf.

2.- Androphy E. Virus del papiloma humano y verrugas. En: Schaechter: Mecanismos de las enfermedades microbianas. Wolters Kluwer Health; 2013. p. 411-8. http://reubenbooks. co.uk/296845-SPPYAXENOA/.

3.- Teyssié A, Picconi M, Alonio V. Virus Papiloma
Humano. En: Microbiología Biomédica. Basualdo JA, Coto CE, de Torres R A., editores. 2a ed. Buenos Aires: Atlante; 2006. p. 798-808.

4.- OPS/OMS | Cáncer Cervicouterino [Internet]. [citado 17 de septiembre de 2019]. Disponible en: https://www.paho. org/hq/index.php?option=com_content \&view=article\&id=5420:2018-cervicalcancer\&Itemid=3637\&lang $=$ es. 
5.- $\mathrm{WHO} \mid$ Cervical cancer [Internet]. WHO World Health Organization; 2018 [citado 17 de septiembre de 2019]. Disponible en: https:// www.who.int/cancer/prevention/diagnosisscreening/cervical-cancer/en/

6.- Ministerio de Salud y Desarrollo Social. Argentina.gob.ar [Internet]. [citado 19 de junio de 2019]. Disponible en: https://www.argentina. gob.ar/salud/instituto-nacional-del-cancer/ estadisticas/incidencia.

7.- Picconi M, Teyssié A. Papilomavirus humanos. En: Virología Médica. 4a ed. Buenos Aires: Corpus; 2015. p. 589-98.

8.- Gupta S, Gupta S. Role of human papillomavirus in oral squamous cell carcinoma and oral potentially malignant disorders: A review of the literature. Indian J Dent 2015; 6 (2): 91-8. doi: 10.4103/0975-962X.155877.

9.- Ellenson L, Pirog E. Aparato genital femenino. En: Robbins y Cotran Patología estructural y funcional. Elsevier; 2010. p. 1005-64.

10.- Li S, Shen H, Li J, Hou X, Zhang K, Li J. Prevalence of the integration status for human papillomavirus 16 in esophageal carcinoma samples. Turkish J Gastroenterol 2018; 29 (2): 157-63. doi: 10.5152/tjg.2018.17568.

11.- Manos M, Ting Shin Y, Wright D, Lewis A, Broker T, Wolinsky S, et al. The use of polymerase chain reaction amplification for the detection of genital human papillomaviruses. 1989; 7: 209-14. https://www.scienceopen. com/document?vid=9ec5d1ec-05b8-486f-aa20$7279 b 7 a d d 122$

12.- Bernard H-U, Chan S-Y, Manos M M, Ong C-K, Villa L L, Delius H, et al. Identification and assessment of known and novel human papillomaviruses by polymerase chain reaction amplification, restriction fragment length polymorphisms, nucleotide sequence, and phylogenetic algorithms. J Infect Dis 1994; 170 (5): 1077-85. doi: 10.1093/ infdis/170.5.1077.

13.- Bruni L, Díaz M, Castellsagué X, Ferrer E,
Bosch F X, de Sanjosé S. Cervical human papillomavirus prevalence in 5 continents: Meta-analysis of 1 million women with normal cytological findings. J Infect Dis 2010; 202 (12): 1789-99. doi: 10.1086/657321.

14.- Sotlar K, Diemer D, Dethleffs A, Hack Y, Stubner A, Vollmer N, et al. Detection and typing of human papillomavirus by E6 nested multiplex PCR. J Clin Microbiol 2004; 42 (7): 3176-84. PMID: 15243079.

15.- Camargo Pinzón S. Determinación de la prevalencia de infección y coinfección por virus del papiloma humano (VPH) y asociación con diferentes factores de riesgo. Universidad del Rosario; 2011. https://repository.urosario.edu. co/handle/10336/2666.

16.- Tonón S, Picconi M, Zinovich J, Nardari W, Mampaey M, Galuppo J, et al. Prevalencia de la infección cervical por virus papiloma humano (HPV) en población caucásica y guaraní, residente en la provincia de Misiones, Argentina. Rev Argent Microbiol 2003; 35 (4): 205-13. PMID: 14976873.

17.- Badano I, Pedrozo R W, Ruíz Díaz L S, Galuppo J A, Picconi M A, Campos R H, et al. Human papillomavirus (HPV) detection and Papanicolaou cytology in low-resource women in Posadas city, Misiones, Argentina Rev Argent Microbiol 2011; 43 (4): 263-7. doi:10.1590/S0325-75412011000400005.

18.- Venezuela R F, Kiguen A X, Frutos M C, Cuffini C G. Circulation of human papillomavirus (HPV) genotypes in women from Córdoba, Argentina, with squamous intraepithelial lesions. Rev Inst Med Trop Sao Paulo 54 (1): 11-6. doi:10.1590/s003646652012000100003.

19.- Deluca G D, Alonso J M. Chlamydia trachomatis and papillomavirus infection in women with cytohistological abnormalities in uterine cervix. Medicina (B Aires) 2006; 66: 303-6. PMID: 16977964.

20.- Golfetto L, Alves E V, Martins T R, Sincero T
C M, Castro J B S, Dannebrock C, et al. PCR-RFLP assay as an option for primary HPV test. Brazilian J Med Biol Res 2018; 51(5):e7098. doi: 10.1590/1414$431 X 20177098$.

21.- Clifford G, Gallus S, Herrero R, Muñoz N, Snijders P, Vaccarella S, et al. Worldwide distribution of human papillomavirus types in cytologically normal women in the International Agency for Research on Cancer HPV prevalence surveys: a pooled analysis. Lancet 2005; 366 (9490): 991-8. doi:10.1016/ S0140-6736(05)67069-9.

22.- Mendoza L, Arbiza J, Páez M, Kasamatsu E, Castro A, Giménez G, et al. Características clínico-demográficas y tipificación del virus de papiloma humano en mujeres paraguayas con citologías negativas para lesión escamosa intraepitelial. Memorias del Inst Investig en Ciencias la Salud. 9 de junio de 2012; 10 (1): 46-55. https://revistascientificas.una.py/index. $\mathrm{php} / \mathrm{RIIC} /$ article/view/136/76.

23.- Toro de Méndez M, López de Sánchez M. Infección por virus papiloma humano en pacientes con citología de cuello uterino negativa. Rev Obstet Ginecol Venez. 2017; 77 (1): 11-20. http://ve.scielo.org/pdf/og/v77n1/ art03.pdf.

24.- Medina M, Medina M, Merino L. Principales conductas de riesgo sobre papilomavirus humano en universitarios argentinos. Av Odontoestomatol. 2018; 34(6): 311-9. http:// scielo.isciii.es/pdf/odonto/v34n6/0213-1285odonto-34-6-311.pdf.

25.- Jaramillo Koupermann G, Ponce Coello T M, Uyaguari Díaz L M. Prevalencia de infección por genotipos de HPV de alto riesgo establecida por captura híbrida y su relación con lesiones diplásicas de cuello uterino, establecidas mediante citología convencional en trabajadoras de la salud. Quito 2014. Quito: UCE; 2015. http://www.dspace.uce.edu.ec/ handle/25000/4634. 